\title{
TITLE:
}

\section{Ultrasonic evaluation of interlayer interfacial stiffness of multilayered structures}

AUTHOR(S):

Ishii, Yosuke; Biwa, Shiro

CITATION:

Ishii, Yosuke ... [et al]. Ultrasonic evaluation of interlayer interfacial stiffness of multilayered structures. Journal of Applied Physics 2012, 111(8): 084907.

ISSUE DATE:

2012-04

URL:

http://hdl.handle.net/2433/155802

RIGHT:

(C) 2012 American Institute of Physics. 


\section{AIP Appolied Physics}

\section{Ultrasonic evaluation of interlayer interfacial stiffness of multilayered structures}

Yosuke Ishii and Shiro Biwa

Citation: J. Appl. Phys. 111, 084907 (2012); doi: 10.1063/1.4704692

View online: http://dx.doi.org/10.1063/1.4704692

View Table of Contents: http://jap.aip.org/resource/1/JAPIAU/v111/i8

Published by the American Institute of Physics.

\section{Related Articles}

Response to "Comment on 'Observation of anomalous acoustic phonon dispersion in $\mathrm{SrTiO} 3$ by broadband stimulated Brillouin scattering'”[Appl. Phys. Lett. 100, 206101 (2012)]

Appl. Phys. Lett. 100, 206102 (2012)

Interaction of laser generated ultrasonic waves with wedge-shaped samples

Appl. Phys. Lett. 100, 184102 (2012)

Imaging a spatially confined photoacoustic source defined by a distribution of plasmonic nanoparticles J. Appl. Phys. 111, 094305 (2012)

Enlargement of a locally resonant sonic band gap by using double-sides stubbed phononic plates Appl. Phys. Lett. 100, 123506 (2012)

Acoustic spin pumping: Direct generation of spin currents from sound waves in Pt/Y3Fe5O12 hybrid structures J. Appl. Phys. 111, 053903 (2012)

\section{Additional information on J. Appl. Phys.}

Journal Homepage: http://jap.aip.org/

Journal Information: http://jap.aip.org/about/about_the_journal

Top downloads: http://jap.aip.org/features/most_downloaded

Information for Authors: http://jap.aip.org/authors

\section{ADVERTISEMENT}

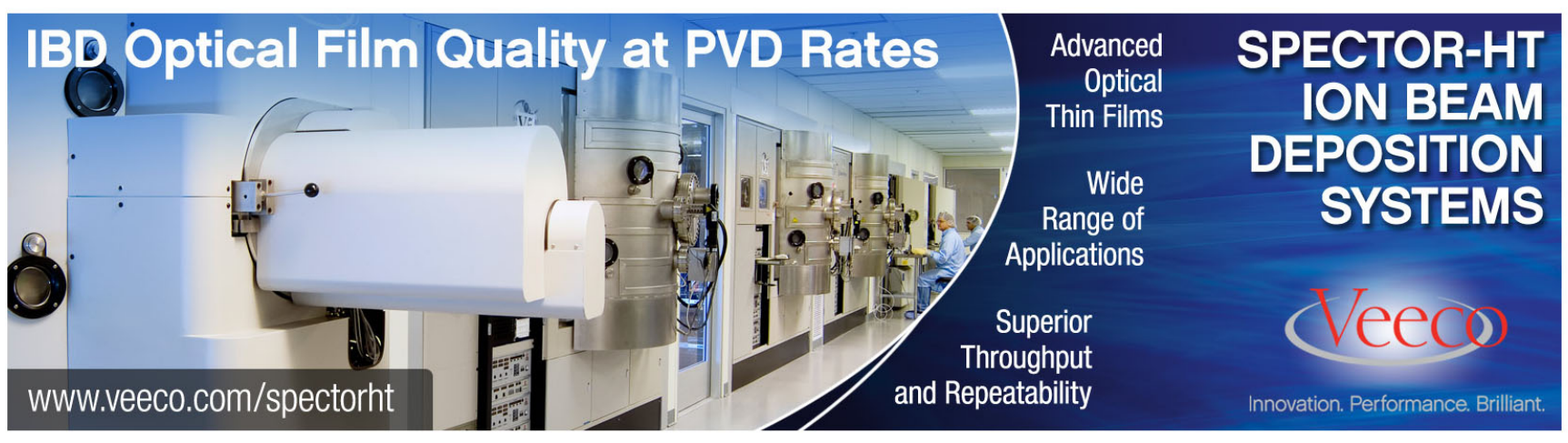




\title{
Ultrasonic evaluation of interlayer interfacial stiffness of multilayered structures
}

\author{
Yosuke Ishii and Shiro Biwa ${ }^{\text {a) }}$ \\ Department of Aeronautics and Astronautics, Graduate School of Engineering, Kyoto University, \\ Yoshida-honmachi, Sakyo-ku, Kyoto 606-8501, Japan
}

(Received 8 January 2012; accepted 16 March 2012; published online 24 April 2012)

\begin{abstract}
A procedure for the ultrasonic evaluation of the interlayer interfacial stiffness of multilayered structures is proposed. As a theoretical background to this proposal, the elastic wave propagation in a multilayered structure, in which the layers are bonded with spring-type interfaces, is analyzed theoretically based on the transfer-matrix method. Using the notion of the Bloch phase which characterizes wave transmission in the corresponding infinite periodic structure, some explicit relations are derived for the reflection coefficient of the multilayered structure. Based on the features clarified theoretically, the interlayer interfacial stiffness of the multilayered structure can be evaluated from the locations of local minima and maxima of the amplitude reflection spectrum. By numerical analysis, the proposed procedure is shown to apply even when the viscous property of the layers is not known precisely, and when a transient waveform of a limited length is used. Using the proposed procedure, the stiffness of interlayer resin-rich regions in a carbon-epoxy cross-ply composite laminate is identified from the experimental reflection spectrum. The identified stiffness is shown to lie within the range as expected from the micrographic observation and a simple estimate for a thin resin layer. (C) 2012 American Institute of Physics. [http://dx.doi.org/10.1063/1.4704692]
\end{abstract}

\section{INTRODUCTION}

Multilayered structures are commonly found in different fields of technological applications. Such structures include, for example, fiber-reinforced composite laminates which are widely used in aerospace engineering. Understanding of elastic wave propagation characteristics in multilayered structures is important regarding their design against dynamic loading as well as their nondestructive diagnosis using ultrasonic waves. This subject has been studied extensively, ${ }^{1,2}$ and various analytical approaches such as the transfer-matrix method ${ }^{3,4}$ have been developed. For periodic layered structures, the so-called Floquet wave homogenization enables the clarification of the dispersive nature of the propagating wave and the existence of stop bands. ${ }^{5-7}$

When modeling wave propagation in multilayered structures either theoretically or numerically, it is important to account for the nature of interfaces between adjacent layers. Many types of interfacial imperfections can be found in different layered structures, such as thin interphase layers, weak bonds, delaminated but contacting surfaces, and so on. In the case of fiber-reinforced composite laminates, it is well known that thin resin-rich regions, typically with thickness of a few microns, exist between laminas. Foregoing studies $^{8-11}$ have revealed that such imperfect interfaces are most simply modeled as spring-type interfaces, where the stresses are continuous but the displacements suffer discontinuities. These interfaces are hence characterized by their normal and tangential stiffnesses.

In order to perform the analysis or numerical simulations of elastic wave propagation for actual multilayered structures,

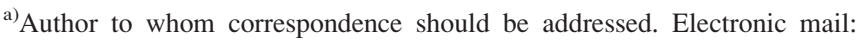
biwa@kuaero.kyoto-u.ac.jp.
}

one needs to identify their interfacial stiffnesses experimentally as material properties. ${ }^{7,12}$ For a single spring-type interface between solid bodies, the ultrasonic reflection/transmission coefficients ${ }^{13-15}$ or the wave velocity and attenuation along the interface $^{16-18}$ can yield the interfacial stiffnesses. For double spring-type interfaces for a layer between solids, Lavrentyev and Rokhlin ${ }^{19}$ proposed a technique to identify the interfacial stiffness from the ultrasonic reflection spectrum. For multiple spring-type interfaces in multilayered structures, $\mathrm{Lu}$ and Achenbach ${ }^{20}$ and $\mathrm{Lu}^{21}$ analyzed the wave propagation behavior based on the transfer-matrix technique and examined the influence of random variations of the interfacial stiffness. Many other works have been carried out for ultrasonic characterization of the interfacial weakness in multilayered structures. ${ }^{22,23}$ Due to the complex wave propagation behavior in multilayered systems, however, it is not a straightforward task to identify the interfacial stiffness from a limited number of observations.

In this paper, the elastic wave propagation in multilayered structures with spring-type interfaces is explored theoretically, and the relation between the interfacial stiffness and the reflection spectrum is investigated. This leads to the proposal of an identification procedure for the interlayer interfacial stiffness of multilayered structures by utilizing the oscillatory nature of the reflection spectrum. Some numerical simulations and experimental results are shown to demonstrate the identification procedure for a carbon-epoxy crossply composite laminate.

This paper is structured as follows. The one-dimensional wave propagation in a multilayered structure with spring-type interfaces is considered based on the transfer-matrix approach in Sec. II. The so-called Bloch phase, ${ }^{24,25}$ which characterizes the wave nature in infinitely repeated periodic structures, is also closely related to the nature of the transfer matrix for a finite 
number of interfaces, and gives some explicit relations for the reflection coefficient of the multilayer. In particular, it is shown that the locations of the zeroes of the amplitude reflection spectrum are completely determined by the acoustic properties of the layer and the interfacial stiffness. Based on the described features, a procedure to identify the interfacial stiffness from local minima and maxima of the reflection spectrum is proposed in Sec. III, and some discussions are given on its feasibility. In Sec. IV, quantitative experimental results for a cross-ply laminate of carbon-epoxy composite are also demonstrated. The conclusions of the present study are summarized in Sec. V.

\section{WAVE PROPAGATION IN A MULTILAYERED STRUCTURE WITH SPRING-TYPE INTERFACES}

\section{A. Transfer matrix formulation}

The multilayered structure to be considered has $N$ elastic layers of density $\rho$, wave velocity $c$, and thickness $h$, with $(N-1)$ spring-type interfaces of stiffness $K_{\mathrm{S}}$ as shown in Fig. 1. The structure is acoustically coupled to semi-infinite media (e.g., water in the case of immersion testing) of density $\rho_{0}$ and velocity $c_{0}$ occupying $x<X_{0}$ and $x>X_{N}$, and subjected to longitudinal wave propagation in the $x$-direction normal to the layers. The interlayer interfaces are located at $x=X_{1}, X_{2}, \ldots, X_{N-1}$. Since all layers are assumed to have the same thickness, $X_{J}=X_{J-1}+h$ for $J=1,2, \ldots, N$.

The harmonic wave propagation with angular frequency $\omega$ is considered (the temporal dependence of the form $\exp (-i \omega t)$ is assumed), and the wave displacement is denoted as $U(x)$. The wave field in each layer consists of the forward-propagating wave $U_{\mathrm{F}}(x)$ and the backwardpropagating wave $U_{\mathrm{B}}(x)$, i.e., $U(x)=U_{\mathrm{F}}(x)+U_{\mathrm{B}}(x)$.

The values of $U_{\mathrm{F}}$ and $U_{\mathrm{B}}$ at the left side of the interface at $x=X_{J}$, i.e., when one approaches this interface from $x<X_{J}$, are denoted by $U_{F}^{J,-}$ and $U_{B}^{J,-}$, respectively. Likewise, $U_{F}^{J,+}$ and $U_{F}^{J,+}$ denote the waves at the right side of that interface. The transfer-matrix method ${ }^{3,4}$ can be used to express the wave transmission across the layered structure. First, for $J=1,2, \ldots, N$, the waves on both sides of the layer at $X_{J-1}<x<X_{J}$ are connected as

$$
\left(\begin{array}{c}
U_{\mathrm{F}}^{J,-} \\
U_{\mathrm{B}}^{J,-}
\end{array}\right)=\mathbf{P}\left(\begin{array}{c}
U_{\mathrm{F}}^{J-1,+} \\
U_{\mathrm{B}}^{J-1,+}
\end{array}\right), \quad \mathbf{P}=\left[\begin{array}{cc}
\exp (i k h) & 0 \\
0 & \exp (-i k h)
\end{array}\right]
$$

where $k=\omega / c$. From the spring-type boundary conditions, ${ }^{20}$ the waves on both sides of the interlayer interface at $x=X_{J}$ $(J=1,2, \ldots \ldots, N-1)$ are related by

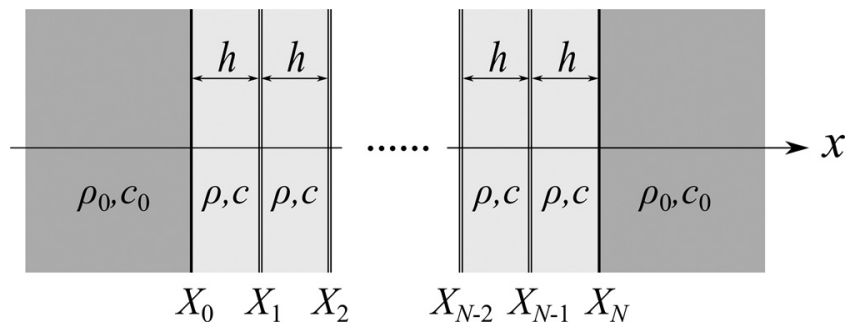

FIG. 1. A multilayered structure with spring-type interfaces.

$$
\left(\begin{array}{c}
U_{\mathrm{F}}^{J,+} \\
U_{\mathrm{B}}^{J,+}
\end{array}\right)=\mathbf{S}\left(\begin{array}{c}
U_{\mathrm{F}}^{J,-} \\
U_{\mathrm{B}}^{J,-}
\end{array}\right), \quad \mathbf{S}=\left[\begin{array}{cc}
1+i \alpha & -i \alpha \\
i \alpha & 1-i \alpha
\end{array}\right]
$$

where

$$
\alpha=\frac{k \rho c^{2}}{2 K_{\mathrm{S}}}=\frac{\rho c \omega}{2 K_{\mathrm{S}}} .
$$

Furthermore, the perfect acoustical coupling (continuity of displacement and stress) at $x=X_{0}$ and $x=X_{N}$ gives

$$
\left(\begin{array}{c}
U_{\mathrm{F}}^{0,+} \\
U_{\mathrm{B}}^{0,+}
\end{array}\right)=\mathbf{T}_{\mathrm{WL}}\left(\begin{array}{c}
U_{\mathrm{F}}^{0,-} \\
U_{\mathrm{B}}^{0,-}
\end{array}\right),\left(\begin{array}{c}
U_{\mathrm{F}}^{N,+} \\
U_{\mathrm{B}}^{N,+}
\end{array}\right)=\mathbf{T}_{\mathrm{LW}}\left(\begin{array}{c}
U_{\mathrm{F}}^{N,-} \\
U_{\mathrm{B}}^{N,-}
\end{array}\right),
$$

where

$$
\mathbf{T}_{\mathrm{WL}}=\frac{1}{2 Z}\left[\begin{array}{cc}
Z+Z_{0} & Z-Z_{0} \\
Z-Z_{0} & Z+Z_{0}
\end{array}\right], \mathbf{T}_{\mathrm{LW}}=\frac{1}{2 Z_{0}}\left[\begin{array}{cc}
Z+Z_{0} & Z_{0}-Z \\
Z_{0}-Z & Z+Z_{0}
\end{array}\right],
$$

by denoting the characteristic acoustic impedances as $Z=\rho c$ and $Z_{0}=\rho_{0} c_{0}$.

Therefore, the whole transmission through the multilayer is expressed by

$$
\left(\begin{array}{c}
U_{\mathrm{F}}^{N,+} \\
U_{\mathrm{B}}^{N,+}
\end{array}\right)=\mathbf{L}\left(\begin{array}{c}
U_{\mathrm{F}}^{0,-} \\
U_{\mathrm{B}}^{0,-}
\end{array}\right)
$$

where

$\mathbf{L}=\left[\begin{array}{ll}L_{11} & L_{12} \\ L_{21} & L_{22}\end{array}\right]=\mathbf{T}_{\mathrm{LW}} \mathbf{P}(\mathbf{S P})^{N-1} \mathbf{T}_{\mathrm{WL}}=\mathbf{T}_{\mathrm{LW}} \mathbf{S}^{-1}(\mathbf{S P})^{N} \mathbf{T}_{\mathrm{WL}}$

If an incident wave with unit amplitude impinges on the structure in Fig. 1, it generates the reflected wave for $x<X_{0}$ and the transmitted wave for $x>X_{N}$, and the waves at $x=X_{0}$ and at $x=X_{N}$ can be written as

$$
\begin{aligned}
& \left(\begin{array}{c}
U_{\mathrm{F}}^{0,-} \\
U_{\mathrm{B}}^{0,-}
\end{array}\right)=\left(\begin{array}{c}
\exp \left(i k_{0} X_{0}\right) \\
R \exp \left(-i k_{0} X_{0}\right)
\end{array}\right), \\
& \left(\begin{array}{c}
U_{\mathrm{F}}^{N,+} \\
U_{\mathrm{B}}^{N,+}
\end{array}\right)=\left(\begin{array}{c}
T \exp \left(i k_{0} X_{N}\right) \\
0
\end{array}\right),
\end{aligned}
$$

where $k_{0}=\omega / c_{0}$, and $R$ and $T$ are the reflection and transmission coefficients, respectively. From Eqs. (6) and (8), $R$ and $T$ are given by

$$
\begin{aligned}
& R=-\frac{L_{21}}{L_{22}} \exp \left(2 i k_{0} X_{0}\right), \\
& T=\frac{L_{11} L_{22}-L_{12} L_{21}}{L_{22}} \exp \left\{-i k_{0}\left(X_{N}-X_{0}\right)\right\} .
\end{aligned}
$$

\section{B. Bloch phase and zeroes of reflection coefficient}

For the present discussion, it is convenient to introduce the following matrix $\mathbf{H}$ and write its components as 


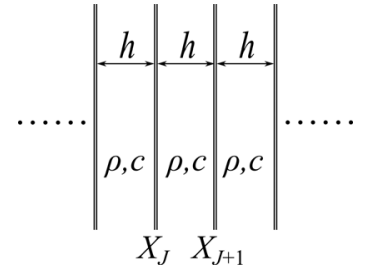

FIG. 2. An infinite periodic structure with spring-type interfaces.

$$
\begin{aligned}
\mathbf{H} & \equiv \mathbf{S P}=\left[\begin{array}{ll}
H_{11} & H_{12} \\
H_{21} & H_{22}
\end{array}\right] \\
& =\left[\begin{array}{cc}
(1+i \alpha) \exp (i k h) & -i \alpha \exp (-i k h) \\
i \alpha \exp (i k h) & (1-i \alpha) \exp (-i k h)
\end{array}\right],
\end{aligned}
$$

from Eqs. (1) and (2). It is then straightforward to observe the following relations:

$$
\begin{gathered}
H_{11} H_{22}-H_{12} H_{21}=1, \\
H_{11}+H_{22}=2\{\cos (k h)-\alpha \sin (k h)\}=2 G\left(\omega^{*}\right),
\end{gathered}
$$

where

$$
\omega^{*}=\frac{\omega h}{c}, \quad G\left(\omega^{*}\right) \equiv \cos \omega^{*}-\Lambda \omega^{*} \sin \omega^{*}
$$

In the above expression, $\omega^{*}$ is the non-dimensional frequency, and the non-dimensional parameter,

$$
\Lambda \equiv \frac{\alpha}{k h}=\frac{\rho c^{2}}{2 K_{\mathrm{S}} h},
$$

represents the relative magnitude of the stiffness of the layer against that of the interface.

The nature of the matrix $\mathbf{H}$ is closely related to the socalled Bloch phase, which characterizes the wave propagation in a perfectly periodic structure made of infinitely arranged spring-type interfaces as shown in Fig. 2. For such periodic systems, the Bloch theorem yields

$$
\left(\begin{array}{c}
U_{\mathrm{F}}^{J+1,+} \\
U_{\mathrm{B}}^{J+1,+}
\end{array}\right)=\exp (i K h)\left(\begin{array}{c}
U_{\mathrm{F}}^{J,+} \\
U_{\mathrm{B}}^{J,+}
\end{array}\right)
$$

where $K$ is the Bloch wave number and $\beta \equiv K h$ is called the Bloch phase. ${ }^{24,25}$ On the other hand, Eqs. (1) and (2) give

$$
\left(\begin{array}{c}
U_{\mathrm{F}}^{J+1,+} \\
U_{\mathrm{B}}^{J+1,+}
\end{array}\right)=\mathbf{S P}\left(\begin{array}{c}
U_{\mathrm{F}}^{J,+} \\
U_{\mathrm{B}}^{J,+}
\end{array}\right)=\mathbf{H}\left(\begin{array}{c}
U_{\mathrm{F}}^{J,+} \\
U_{\mathrm{B}}^{J,+}
\end{array}\right) .
$$

From Eqs. (15) and (16), it is clear that $\exp (i K h)$ is an eigenvalue of the matrix $\mathbf{H}$, i.e.,

$$
\{\exp (i \beta)\}^{2}-\left(H_{11}+H_{22}\right) \exp (i \beta)+H_{11} H_{22}-H_{12} H_{21}=0 .
$$

This condition gives, by aid of Eqs. (11) and (12),

$$
\exp (i \beta)=G\left(\omega^{*}\right) \pm i \sqrt{1-\left\{G\left(\omega^{*}\right)\right\}^{2}}
$$

When $-1 \leq G\left(\omega^{*}\right) \leq 1$, Eq. (18) determines $\beta$ as a real value given by

$$
\cos \beta=G\left(\omega^{*}\right),
$$

which gives the dispersion relation in the pass bands of the infinite periodic structure. Furthermore, in this case the Cayley-Hamilton theorem gives

$$
\mathbf{H}^{2}-2 \cos \beta \mathbf{H}+\mathbf{I}=0,
$$

using Eqs. (11), (12), and (19). Then a mathematical induction yields ${ }^{24}$

$$
\mathbf{H}^{N}=\frac{\sin (N \beta)}{\sin \beta} \mathbf{H}-\frac{\sin \{(N-1) \beta\}}{\sin \beta} \mathbf{I} .
$$

When $\left|G\left(\omega^{*}\right)\right|>1$, however, $\beta$ becomes complex, giving stop bands of the infinite periodic structure. In this case, $\operatorname{Re}[\beta]=m \pi$, where the integer $m$ is determined so as to make the dispersion curve continuous.

It is important to note here that while the Bloch phase $\beta$ is originally a parameter to characterize the wave in the infinite periodic structure in Fig. 2, it is also connected closely to the nature of the matrix $\mathbf{H}$, and further to the wave propagation in the finite multilayered structure in Fig. 1. Based on the mathematical derivation given above, the relation between the angular frequency $\omega$ and the Bloch phase $\beta$ can be made clear, as illustrated in Fig. 3. When $\omega^{*}$ increases from $0, G\left(\omega^{*}\right)$ decreases from 1 and $\beta$ increases from 0 . At a certain $\omega^{*}$, $G\left(\omega^{*}\right)$ becomes -1 and $\beta=\pi$. Beyond this point, $G\left(\omega^{*}\right)$ becomes less than -1 and $\beta$ becomes a complex value, where the pass-band terminates and the first stop-band starts in the case of periodic structure. These relationships are shown in

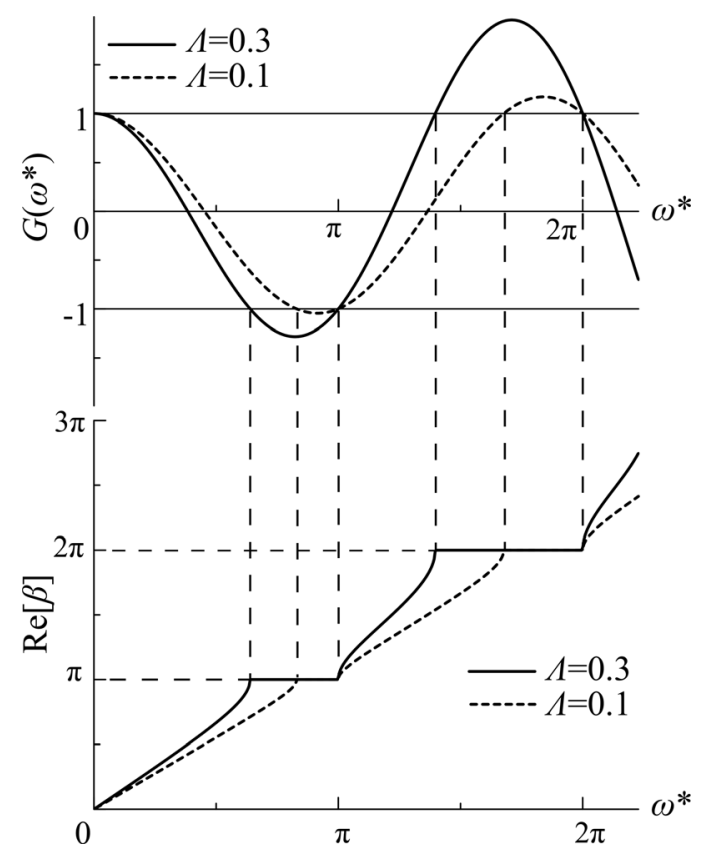

FIG. 3. The relation between the nondimensional frequency and the Bloch phase for different $\Lambda$. 
Fig. 3 for different values of $\Lambda$, which indicates that a wider stop band appears for a greater $\Lambda$.

Attention is now turned to the reflection coefficient of the finite multilayered structure. Using Eqs. (7) and (21), the matrix $\mathbf{L}$ is rewritten as

$$
\mathbf{L}=\frac{\sin (N \beta)}{\sin \beta} \mathbf{T}_{\mathrm{LW}} \mathbf{P} \mathbf{T}_{\mathrm{WL}}-\frac{\sin \{(N-1) \beta\}}{\sin \beta} \mathbf{T}_{\mathrm{LW}} \mathbf{S}^{-1} \mathbf{T}_{\mathrm{WL}}
$$

Explicit computation of the right-hand side of the above expression gives

$$
\frac{L_{21}}{L_{22}}=\frac{\left(\zeta^{2}-1\right) \sin (N \beta) \sin \omega^{*}-2 \Lambda \omega^{*} \sin \{(N-1) \beta\}}{\left(\zeta^{2}+1\right) \sin (N \beta) \sin \omega^{*}+2 \Lambda \omega^{*} \sin \{(N-1) \beta\}+2 i \zeta\left[\sin (N \beta) \cos \omega^{*}-\sin \{(N-1) \beta\}\right]},
$$

where

$$
\zeta=\frac{Z}{Z_{0}}
$$

In the pass bands, the numerator of the right-hand side of Eq. (23) is real and can be written as

$$
\begin{aligned}
& \left(\zeta^{2}-1\right) \sin (N \beta) \sin \omega^{*}-2 \Lambda \omega^{*} \sin \{(N-1) \beta\} \\
& =\sqrt{A^{2}+B^{2}} \sin (N \beta+\gamma),
\end{aligned}
$$

where $A, B$, and $\gamma$ are given by

$$
\begin{gathered}
A=\left(\zeta^{2}-1\right) \sin \omega^{*}-2 \Lambda \omega^{*} \cos \beta, \quad B=2 \Lambda \omega^{*} \sin \beta, \\
\cos \gamma=\frac{A}{\sqrt{A^{2}+B^{2}}}, \quad \sin \gamma=\frac{B}{\sqrt{A^{2}+B^{2}}} .
\end{gathered}
$$
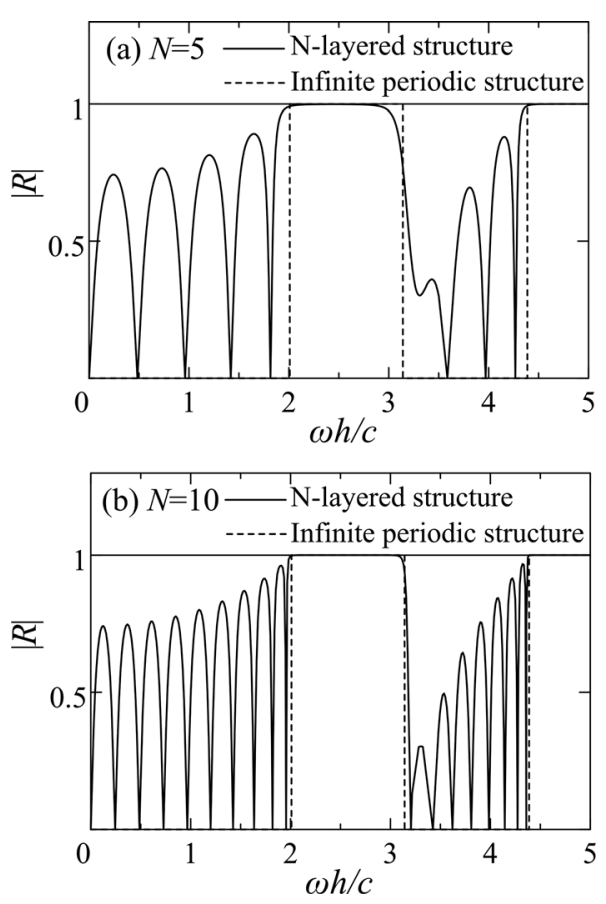

FIG. 4. The amplitude reflection spectrum of the multilayered structure for $\zeta=3.3$ and $\Lambda=0.3$, (a) $N=5$ and (b) $N=10$, together with the reflection spectrum of the corresponding infinite periodic structure.

Namely, Eq. (23), hence the reflection coefficient $R$ in Eq. (9), vanishes when

$$
N \beta+\gamma=n \pi
$$

where $n$ is an integer. The locations of the zeroes of the reflection coefficient on the $\beta$-axis or on the $\omega^{*}$-axis are determined by Eqs. (13), (14), and (28). It is noted that the number of these zeroes for $0<\beta<\pi$ are governed by the parameters $N, \Lambda$, and $\zeta$.

The variation of the amplitude reflection coefficient $|R|$ with the frequency is illustrated in Figs. 4 and 5 for different $N$ and $\Lambda$ for a fixed $\zeta$. The amplitude spectra of the corresponding infinite periodic structures are also shown for comparison, which exhibit clearly defined pass bands $(|R|=0)$ and stop bands $(|R|=1)$. It is easily recognized
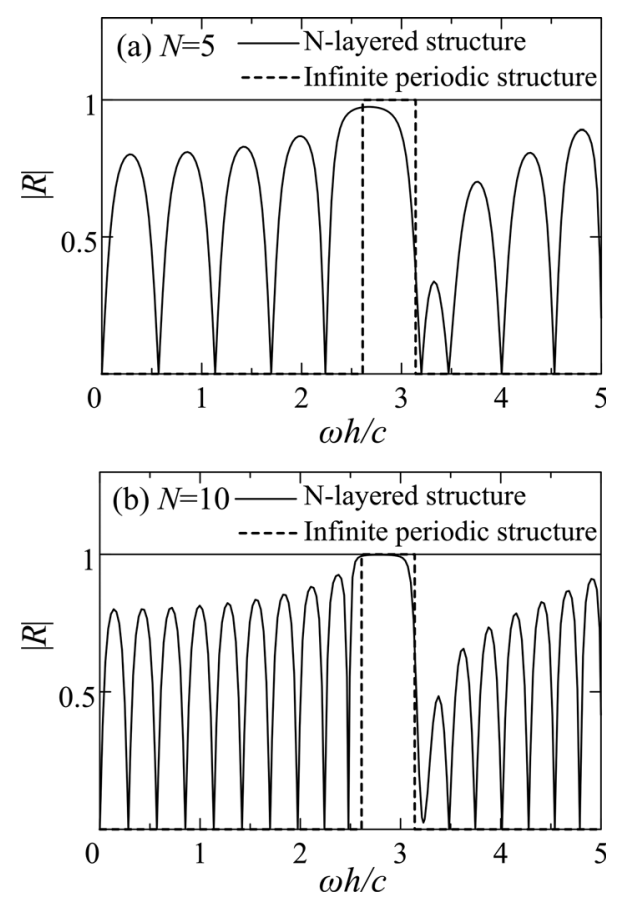

FIG. 5. The amplitude reflection spectrum of the multilayered structure for $\zeta=3.3$ and $\Lambda=0.1$, (a) $N=5$ and (b) $N=10$, together with the reflection spectrum of the corresponding infinite periodic structure. 
in Figs. 4 and 5 that for the chosen set of parameters, the 5-layered (10-layered) structure has 4 zeroes (9 zeroes) (besides $\omega=0$ ) below the first stop band in the infinite periodic structure.

\section{EVALUATION OF INTERLAYER INTERFACIAL STIFFNESS}

\section{A. Proposal for the stiffness evaluation procedure}

The above discussion has shown that the reflection coefficient of the $N$-layered structure takes zeroes at certain discrete points on the frequency axis in the first pass band of the corresponding infinite periodic structure. The locations of these zeroes are influenced by $N, \Lambda$, and $\zeta$, thus by the interfacial stiffness as well as the acoustic properties of the layers. The measurement of these frequencies will then enable the determination of the interfacial stiffness, provided that the acoustic properties of the layers are known. Figures 4 and 5 also show that the amplitude reflection coefficient $|R|$ exhibits local minima (i.e., zeroes) and maxima in the first pass band, which locations are also dependent on the interfacial stiffness. When the reflection spectrum is obtained experimentally, these local extrema are more easily identified than the exact zeroes of the reflection coefficient. In Fig. 6 , these extremum points are depicted as functions of $\Lambda$ for the 16-layered structure with $\zeta=3.3$. It is seen that the variation of the location of these points with $\Lambda$ is monotonic in the first pass band, which makes the determination of $\Lambda$ (hence $K_{\mathrm{S}}$ ) from the measured extremum frequencies relatively straightforward, except in the region of very small $\Lambda$ where all curves tend to be flat.

To summarize, the proposed procedure for the identification of the interfacial stiffness is put forward as follows. The ultrasonic reflection spectrum is measured for a multilayered structure for a sufficiently wide bandwidth, and the extremum frequencies in the experimental reflection spectrum are compared to the theoretical ones in Fig. 6. The value of the interfacial stiffness to give the best fit between the theory and the experiment can then be determined.

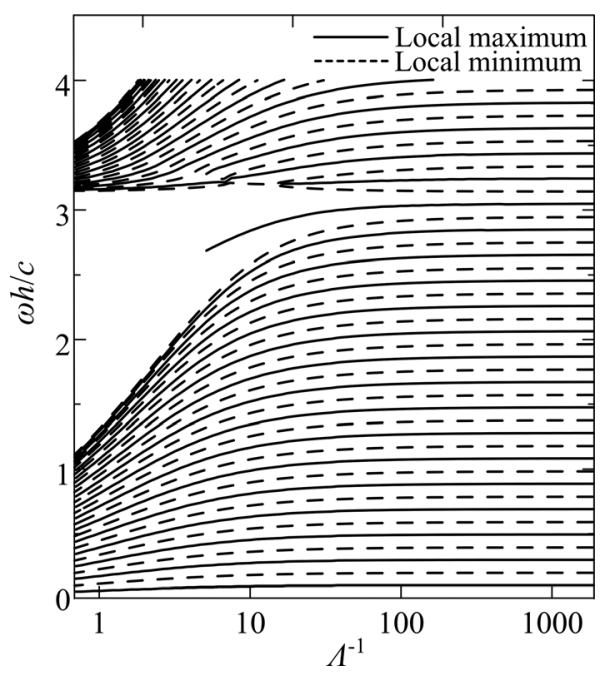

FIG. 6. Variation of the extremum frequencies with $\Lambda$ for $N=16$.

\section{B. Discussions on the proposed procedure}

\section{Influence of viscoelasticity of layers}

Some considerations are made here regarding the feasibility of the proposed procedure from theoretical and numerical points of view. To be specific, consider a carbonfiber-reinforced cross-ply laminate immersed in water, for which an experimental result will be shown in later sections of this paper. For the unidirectional carbon-epoxy composite layer, the acoustic properties in the thickness direction ${ }^{26}$ are shown in Table I, together with those for water. Note that even if unidirectional composite layers are stacked with different angles, the acoustic properties in the stacking direction are the same for all layers, so the composite laminate can be treated just as the multilayered structure discussed above as far as normal-incident longitudinal waves are concerned.

First, it should be remembered that the theoretical discussions in Sec. II are based on the assumption that the layers are elastic. Real carbon-fiber-reinforced layers, however, possess certain viscoelastic nature at ultrasonic frequencies. Therefore, it is necessary to examine if the above procedure is still valid in the presence of the viscoelasticity. To this aim, the layers are modeled as linear viscoelastic solid of Voigt type, whose stress $(\sigma)$-strain $(\varepsilon)$ relation is expressed as

$$
\sigma=C \varepsilon+\eta \frac{\partial \varepsilon}{\partial t}
$$

where $C$ and $\eta$ are the static stiffness and the damping coefficient, respectively. For the composite layer, these parameters are set as shown in Table II from the literature. ${ }^{26}$ In this case, the wave velocity $c$ in the transfer-matrix formulation should be replaced by the frequency-dependent complex velocity,

$$
c_{\mathrm{VE}}(\omega)=\sqrt{\frac{C_{\mathrm{VE}}(\omega)}{\rho}},
$$

where $C_{\mathrm{VE}}(\omega)$ is the complex modulus given by

$$
C_{\mathrm{VE}}(\omega)=C-i \omega \eta
$$

By this replacement, the results in Sec. II A remain valid and the reflection coefficient from the multilayered structure is still given by Eq. (9).

The amplitude reflection spectra for $N=16$ and $K_{\mathrm{S}}=$ $0.2 \mathrm{MPa} / \mathrm{nm}$ are shown in Fig. 7 for the cases where the layers are elastic and where they are viscoelastic. The extremum frequencies are shown in Fig. 8 as functions of the interfacial stiffness for both cases. It is found in Fig. 7 that

TABLE I. Acoustic properties of carbon-epoxy composite layer and water.

\begin{tabular}{lcc}
\hline \hline & $\begin{array}{c}\text { Density } \\
\left(\mathrm{kg} / \mathrm{m}^{3}\right)\end{array}$ & $\begin{array}{c}\text { Longitudinal wave } \\
\text { velocity }(\mathrm{m} / \mathrm{s})\end{array}$ \\
\hline Carbon-epoxy composite & 1600 & 3062 \\
Water & 998 & 1500 \\
\hline \hline
\end{tabular}


TABLE II. Viscoelastic properties of carbon-epoxy composite layer.

\begin{tabular}{lcc}
\hline \hline & $\begin{array}{c}\text { Static } \\
\text { stiffness } C(\mathrm{GPa})\end{array}$ & $\begin{array}{c}\text { Damping } \\
\text { coefficient } \eta(\mathrm{Pa} \mathrm{s})\end{array}$ \\
\hline Carbon-epoxy composite & 15.0 & 14.0 \\
\hline \hline
\end{tabular}

while the values of the local minimum and maximum reflection coefficients do change with the introduced viscoelasticity, the extremum frequencies are essentially unchanged (the average error for each $K_{\mathrm{S}}$ being within $0.05 \%$ in the first pass band), except that they show different behavior in the second pass band at low $K_{\mathrm{S}}$ : with the introduction of the viscoelasticity, some extrema disappear above the stop band when the interfacial stiffness is low, say $K_{\mathrm{S}}<0.2 \mathrm{MPa} / \mathrm{nm}$. This subtlety can be circumvented by using only the extremum points in the first pass band for the evaluation of $K_{\mathrm{S}}$. Consequently, the identification procedure for the interfacial stiffness can be used without the full knowledge of viscoelastic properties of the layers, namely, their elastic properties are sufficient.

\section{Application with transient waveforms}

Another source of concern is that while the above discussions are based on the frequency-domain formulation, actual waveform measurements are commonly made in the time domain, and the waveform of a limited length is processed to obtain the reflection spectrum. In order partly to examine if the procedure works well when a transient reflection waveform of limited length is used, and partly to elucidate the features of temporal waveforms, the numerical analysis is performed for transient wave propagation in the composite laminate using the finite element method. The analysis is based on a two-dimensional elastodynamic analysis code with the concentrated-mass approximation and an explicit time-integration scheme with the Runge-Kutta method. It is designed to model the one-dimensional multilayered structure (16 layers with total thickness $2.16 \mathrm{~mm}$ ) immersed in water, accounting for the viscoelastic proper$\operatorname{ties}^{26}$ of the layers and the spring-type interfaces with 3392 degree of freedom. The wave motion is excited in water at $1 \mathrm{~mm}$ away from the surface of the composite laminate as an amplitude-modulated sinusoidal wave with the center frequency $10 \mathrm{MHz}$. It is noted that while the results of the

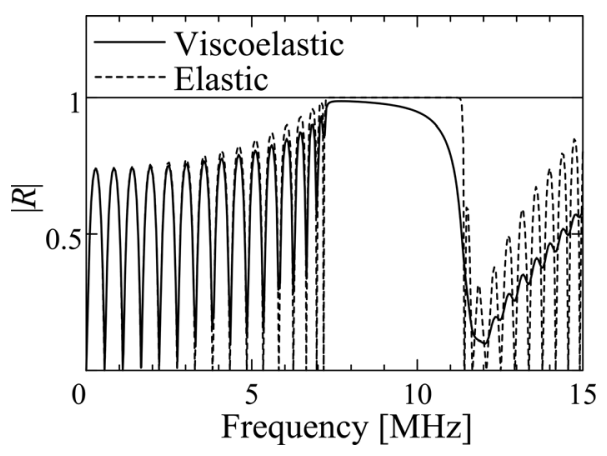

FIG. 7. The amplitude reflection spectrum of the composite laminate when the layers are modeled as elastic and viscoelastic.

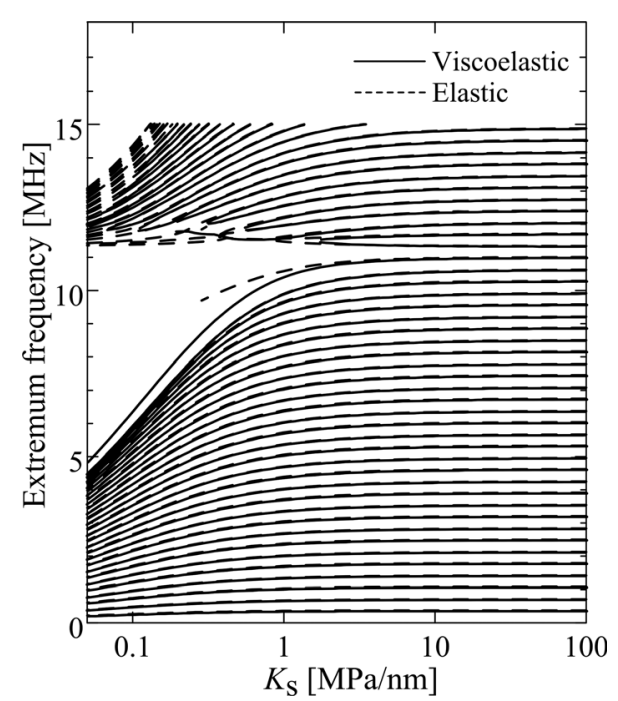

FIG. 8. Variation of the extremum frequencies with the interfacial stiffness when the layers are modeled as elastic and viscoelastic (minima and maxima are not distinguished).

transfer-matrix analysis can be in principle transformed to obtain the time-domain waveforms, the finite element analysis is performed independently here to verify the theoretical analysis.

The computed reflection waveforms are shown in Fig. 9 for different values of the interfacial stiffness. The waveforms show different characteristics according to the variation of the stiffness. Namely, as the interfacial stiffness is reduced, the bottom echo is delayed significantly, and the reverberation just behind the surface echo, due to the reflection at different interlayer interfaces, becomes remarkable. It is noted, however, that only with these features the determination of the interfacial stiffness is difficult.

In the case for $K_{\mathrm{S}}=0.8 \mathrm{MPa} / \mathrm{nm}$, the amplitude reflection spectra are calculated by the fast Fourier transform of the waveform in Fig. 9, gated for different intervals as (i) $0<t<2 \mu \mathrm{s}$, (ii) $0<t<3.5 \mu \mathrm{s}$, and (iii) $0<t<5 \mu \mathrm{s}$. The results are shown in Fig. 10, together with the corresponding spectrum calculated by the transfer-matrix method of

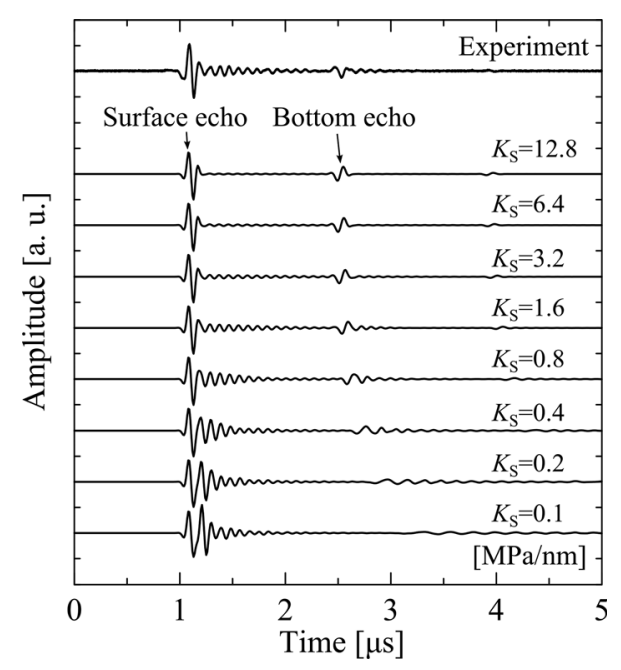

FIG. 9. Temporal reflection waveforms. 


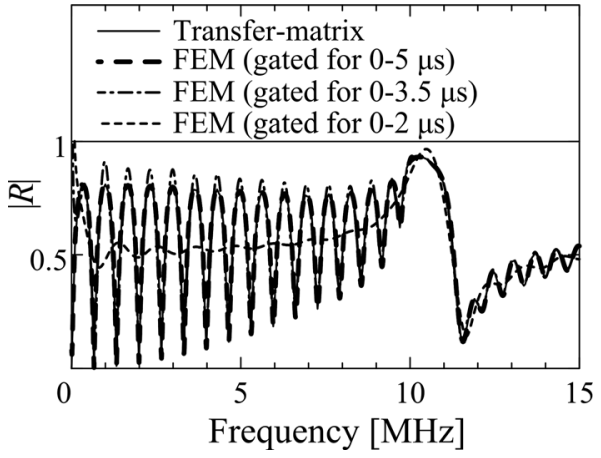

FIG. 10. The amplitude reflection spectrum of the composite laminate as calculated by the finite element analysis and the transfer-matrix method.

Sec. II A in the frequency domain. It is then found that the extremum frequencies from the transient waveforms gated with the intervals (ii) and (iii) are indistinguishable from those of the transfer-matrix method (the errors being within $0.2 \%$ ), while the spectrum for the interval (i) does not provide the required information of local extrema. Therefore, it is considered that the proposed identification procedure for the interfacial stiffness applies for practical experimental arrangements, as long as the waveform is acquired so as to contain from the surface echo up to at least the first bottom echo signals.

\section{APPLICATION TO A CROSS-PLY COMPOSITE LAMINATE}

\section{A. Experimental procedure}

The reflection measurement was performed for a crossply laminate of carbon-fiber-reinforced epoxy composite sample (16 layers, thickness $2.16 \mathrm{~mm}$ ) with the TR30 carbon fibers and the \#340 epoxy resin, supplied by Mitsubishi Rayon Co. Ltd., Japan. The sample was immersed in water, and the ultrasonic pulse-echo measurement was made with a piezoelectric transducer of nominal frequency $15 \mathrm{MHz}$ and a commercial scanning system FlexScan (Insight, Inc., Tokyo), as shown in Fig. 11. Although the simultaneous determination of the acoustic properties of both plies and interfaces is desirable, in the present study it is assumed that the ply properties are known and given as in Table I, so the relation between the extremum frequencies and the interfacial stiffness is given as in Fig. 8.

The acquired reflection waveform is shown in Fig. 9 together with the numerical results. It may be noted that the

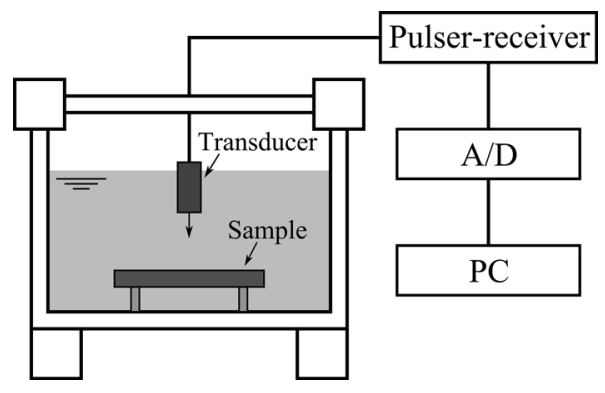

FIG. 11. Experimental setup. reverberation features after the surface echo in the experimental waveform qualitatively resembles one of the computed results with $K_{\mathrm{S}}=1.6 \mathrm{MPa} / \mathrm{nm}$, although substantial ripples are persistent until later time in the experimental waveform due perhaps to uneven interfacial properties or other manufacturing imperfections. Such comparison of waveforms can give a simple estimate of the interfacial stiffness, but its more objective determination is discussed below. The fast Fourier transform of the experimental waveform was obtained, by setting the time-gate as $0<t<5 \mu \mathrm{s}$. In order to calculate the reflection coefficient of the composite laminate, a reference waveform was obtained by measuring the reflected wave from a polished surface of a thick aluminum block immersed in water with the same experimental setting. The amplitude reflection spectrum of the laminate was obtained by dividing its amplitude reflection coefficient by that of the aluminum block at each frequency, also by compensating for the reflection coefficient at the water-aluminum interface which was easily calculated with the acoustic impedances of both media.

\section{B. Results and discussion}

The obtained reflection spectrum of the composite laminate is shown in Fig. 12. The spectrum shows a relatively high peak at around $10.5 \mathrm{MHz}$, in conformity with the numerical results in Fig. 8 corresponding to the first stop band of the corresponding infinite structure. Although the experimental spectrum in Fig. 12 is only shown for a finite frequency range from 5 to $15 \mathrm{MHz}$ because of the limited bandwidth of the measurement, it contains a sufficient number of local maxima and minima. The frequencies of these extremum points are then extracted. It is a relatively a straightforward task to find a best $K_{\mathrm{S}}$ for which these experimental points fit to the theoretical curves, as shown in Fig. 13. This gives the interfacial stiffness as $K_{\mathrm{S}}=1.6$ $\mathrm{MPa} / \mathrm{nm}(\Lambda=0.0347)$, as the value of $K_{\mathrm{S}}$ which minimizes the sum of the squared errors between 17 experimental points in the first pass band and the corresponding theoretical curves. Other extremum frequencies in the second pass band are also depicted in Fig. 13 to demonstrate their agreement with the theoretical curves, although they are not used for the stiffness identification.

Using the so-obtained stiffness, the theoretical reflection spectrum is computed with the transfer-matrix method and

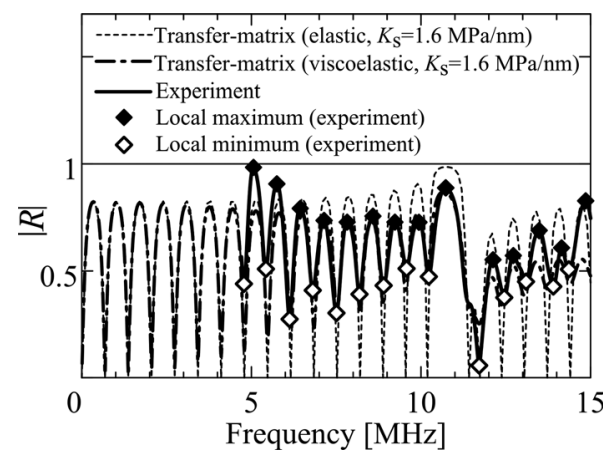

FIG. 12. Experimental and theoretical amplitude reflection spectra of the composite laminate. 


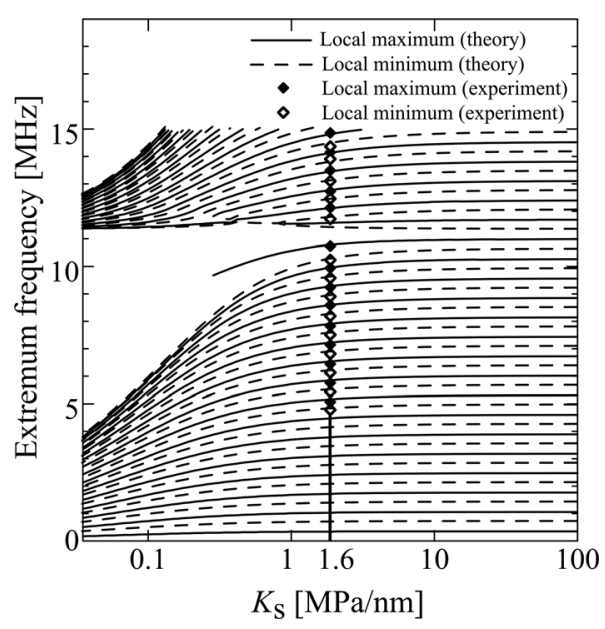

FIG. 13. Experimental extremum frequencies and their theoretical variation with the interfacial stiffness.

shown in Fig. 12 together with the experimental result. The oscillatory characteristics of the reflection coefficient against the frequency near the stop band seen in the experimental result are remarkably well reproduced by the theory.

A micrographic observation was made for the composite laminate sample, and it was confirmed that there were regions between plies occupied only by epoxy resin. These regions were typically 3 to $8 \mu \mathrm{m}$ thick, although they cannot be identified as layers of well-defined thickness. Nevertheless, if these regions are regarded as layers of uniform thickness $h_{\mathrm{e}}$, a simple estimate of their stiffness is given by ${ }^{8,13}$

$$
K_{\mathrm{S}}=\frac{\lambda_{\mathrm{e}}+2 \mu_{\mathrm{e}}}{h_{\mathrm{e}}},
$$

where $\lambda_{\mathrm{e}}$ and $\mu_{\mathrm{e}}$ are Lamé constants of epoxy resin. The value for the epoxy resin from the same supplier as the composite sample, $\lambda_{\mathrm{e}}+2 \mu_{\mathrm{e}}=8.8 \mathrm{GPa},{ }^{27}$ gives estimations of 1.1 to $2.9 \mathrm{MPa} / \mathrm{nm}$ corresponding to the above range of $h_{\mathrm{e}}$. The interfacial stiffness obtained with the proposed procedure is well within this range, which indicates that the estimated value is quite reasonable. In other words, the identified interfacial stiffness implies an equivalent thickness of the resinrich region $h_{\mathrm{e}}=5.5 \mu \mathrm{m}$ based on Eq. (32), which is reasonable in the light of the micrographic observation.

Finally, some comments are made regarding the proposed procedure for the evaluation of the interfacial stiffness $K_{\mathrm{S}}$ from the reflection spectrum. First, in Fig. 6, it is seen that for the region of low $K_{\mathrm{S}}$, the slopes of the curves are sufficiently high so that $K_{\mathrm{S}}$ can be determined with high accuracy. On the other hand, these slopes tend to become very small as $K_{\mathrm{S}}$ becomes higher. This feature may introduce some difficulties in determining the interfacial stiffness from the extremum frequencies.

In order to examine this issue, some perturbation studies have been carried out by numerical analysis. As a result, it has been found that at $K_{\mathrm{S}}=1.6 \mathrm{MPa} / \mathrm{nm}$, a perturbation of 30 $\mathrm{m} / \mathrm{s}$ to the assumed layer wave velocity (amounting to $1 \%$ uncertainty) shifts the estimated $K_{\mathrm{S}}$ by $20 \%$ to $30 \%$, while the $1 \%$ perturbation of the layer mass density merely gives $1 \%$ change of $K_{\mathrm{S}}$. Therefore, it is important to have an accurate layer wave velocity in order to obtain a reliable value of $K_{\mathrm{S}}$. When there are uncertainties in the layer wave velocity, however, the obtained $K_{\mathrm{S}}$ can still serve as a rough estimate which may suffice for practical applications. In the lower range of $K_{\mathrm{S}}$, say at $K_{\mathrm{S}}=0.1 \mathrm{MPa} / \mathrm{nm}$, either $1 \%$ perturbation of the layer wave velocity or the density only causes $1 \%$ variations of the estimated $K_{\mathrm{S}}$, so the present procedure is expected to work well for the evaluation of weakened interfaces.

For the region of even higher $K_{\mathrm{S}}$ where the dependence of the extremum frequencies on $K_{\mathrm{S}}$ is lost, the interlayer interface can be to a good approximation treated as perfectly bonded interface, so the precise determination of $K_{\mathrm{S}}$ may be unnecessary. In fact, when $K_{\mathrm{S}}>10 \mathrm{MPa} / \mathrm{nm}$, say, the extremum frequency curves are nearly flat as shown in Fig. 8. In accordance, the temporal waveform for $K_{\mathrm{S}}=12.8$ $\mathrm{MPa} / \mathrm{nm}$ in Fig. 9 shows that no remarkable internal interface reflections are present, essentially exhibiting characteristics of perfectly bonded layers.

Second, it is noted that the interfacial stiffness has been treated and identified as a frequency-independent real-valued parameter in the present study. When the interlayer interface is an idealization of, for example, a thin resin-rich region as in the composite laminate, the interface not only has a springtype property but also exhibits a certain damping behavior. To characterize the elastic as well as dissipative nature of interlayer interfaces, their stiffness should be evaluated as a possibly frequency-dependent, complex-valued parameter. Among others, Fraisse et al. ${ }^{14}$ identified such features for an adhesive layer between solids. The complex-valued evaluation of interlayer interfacial stiffnesses for multilayered structures is certainly an intriguing subject, but left for the future study.

\section{SUMMARY}

In the present study, the elastic wave propagation in multilayered structures with spring-type interlayer interfaces has been analyzed theoretically based on the transfer-matrix method. Using the notion of the Bloch phase which originally characterizes wave transmission in the corresponding infinite periodic structures, some explicit relations have been derived for the reflection coefficient of the multilayered structure. Based on the features clarified theoretically, a procedure has been proposed to identify the interlayer interfacial stiffness of the multilayered structure from the extremum frequencies in its amplitude reflection spectrum. The proposed procedure has been shown to apply even when the viscous property of the layers is not known and when a transient waveform of a limited length is used. Using the proposed procedure, the stiffness of the interlayer interfaces in a cross-ply composite laminate has been identified from the experimental reflection spectrum, and shown to agree with the range as expected from the micrographic observation and a simple estimate of the stiffness for a thin resin layer. The identified stiffness represents the mechanical property of the interlayer interfaces, and can be used to analyze the wave propagation in the composite laminate. The procedure may be also applicable to other kinds of interlayer interfaces, and is expected to facilitate numerical modeling and nondestructive diagnosis of multilayered structures. 


\section{ACKNOWLEDGMENTS}

The authors acknowledge the kind help offered by Professor M. Hojo and Professor M. Nishikawa for the micrographic observation of the composite sample. The authors are thankful to the anonymous reviewer for the constructive comments on the manuscript.

${ }^{1}$ W. M. Ewing, W. S. Jardetzky, and F. Press, Elastic Waves in Layered Media (McGraw-Hill, New York, 1957).

${ }^{2}$ L. M. Brekhovskikh, Waves in Layered Media (Academic, New York, 1960).

${ }^{3}$ W. T. Thomson, J. Appl. Phys. 21, 89 (1950).

${ }^{4}$ N. A. Haskell, Bull. Seismol. Soc. Am. 43, 17 (1953).

${ }^{5}$ A. H. Nayfeh, J. Acoust. Soc. Am. 89, 1521 (1991).

${ }^{6}$ C. Potel and J.-F. de Belleval, J. Appl. Phys. 74, 2208 (1993).

${ }^{7}$ L. Wang and S. I. Rokhlin, J. Acoust. Soc. Am. 114, 2582 (2003).

${ }^{8}$ A. Boström and G. Wickham, J. Nondestruct. Eval. 10, 139 (1991).

${ }^{9}$ S. I. Rokhlin and Y. J. Wang, J. Acoust. Soc. Am. 89, 503 (1991).

${ }^{10}$ S. I. Rokhlin and W. Huang, J. Acoust. Soc. Am. 92, 1729 (1992).

${ }^{11}$ M. V. Golub, Acoust. Phys. 56, 848 (2010).
${ }^{12}$ B. Hosten and M. Castaings, J. Acoust. Soc. Am. 117, 1108 (2005).

${ }^{13}$ A. Pilarski and J. L. Rose, J. Appl. Phys. 63, 300 (1988).

${ }^{14}$ P. Fraisse, F. Scmit, and A. Zarembowitch, J. Appl. Phys. 72, 3264 (1992).

${ }^{15}$ B. W. Drinkwater, R. S. Dwyer-Joyce, and P. Cawley, Proc. R. Soc. London, Ser. A 452, 2613 (1996).

${ }^{16}$ S. Rokhlin, M. Hefets, and M. Rosen, J. Appl. Phys. 51, 3579 (1980).

${ }^{17}$ B. Drinkwater, M. Castaings, and B. Hosten, J. Acoust. Soc. Am. 113, 3161 (2003).

${ }^{18}$ S. Biwa, S. Hiraiwa, and E. Matsumoto, Ultrasonics 47, 123 (2007).

${ }^{19}$ A. I. Lavrentyev and S. I. Rokhlin, J. Acoust. Soc. Am. 103, 657 (1998).

${ }^{20}$ Y. Lu and J. D. Achenbach, J. Acoust. Soc. Am. 90, 2576 (1991).

${ }^{21}$ Y. Lu, Ultrasonics 30, 289 (1992).

${ }^{22}$ X. M. Jian, N. Guo, M. X. Li, and H. L. Zhang, J. Nondestruct. Eval. 21, 55 (2002).

${ }^{23}$ L. Aïnouche and C. Alquié, J. Appl. Phys. 96, 1475 (2004).

${ }^{24}$ J. M. Bendickson, J. P. Dowling, and M. Scalora, Phys. Rev. E 53, 4107 (1996).

${ }^{25}$ F. Kobayashi, S. Biwa, and N. Ohno, Int. J. Solids Struct. 41, 7361 (2004).

${ }^{26}$ B. Hosten and M. Deschamps, J. Acoust. Soc. Am. 82, 1763 (1987).

${ }^{27}$ S. Biwa, Y. Watanabe, and N. Ohno, Compos. Sci. Technol. 63, 237 (2003). 